\title{
ACAROLOGY
}

\section{Perspectivas para a Criação Massal de Iphiseiodes zuluagai Denmark \& Muma (Acari: Phytoseiidae)}

\author{
FÁbio A. De Albuquerque ${ }^{1}$ e Gilberto J. De Moraes² \\ ${ }^{1}$ Faculdade de Ciências Agrárias e Veterinárias, UNESP - Campus de Jaboticabal, SP. Via de Acesso Prof. Paulo \\ Donato Castellane s/n, 14884-900, Jaboticabal, SP \\ ${ }^{2}$ Escola Superior de Agricultura Luiz de Queiroz, USP - Av. Pádua Dias 11, 13418-900, Piracicaba, SP
}

Neotropical Entomology 37(3):328-333 (2008)

Perspectives for Mass Rearing of Iphiseiodes zuluagai Denmark \& Muma (Acari: Phytoseiidae)

\begin{abstract}
Iphiseiodes zuluagai Denmark \& Muma is an important predator of Brevipalpus phoenicis (Geijskes) on citrus in Brazil. The suitability of Tyrophagus putrescentiae (Schrank) as a food source of $I$. zuluagai in laboratory rearing was investigated at $25.5 \pm 0.5^{\circ} \mathrm{C}, 88 \pm 7 \% \mathrm{RU}$ and photophase of $12 \mathrm{~h}$. Initially, levels of oviposition of the predator fed on eggs were evaluated, as well as the dead or live post-embryonic stages of T. putrescentiae, in a period of 10 days. The daily oviposition rate was 1.3 egg per female when they were fed on eggs on T. putrescentiae, 0.7 egg per female when they were fed on dead post embryonic stages and about 0.3 egg per female when they were fed on live post-embryonic stages. Later, the life table of I. zuluagai was constructed, when eggs of $T$. putrescentiae were offered to the predators as prey. The immature stages were observed every $8 \mathrm{~h}$, to determine the corresponding durations. In the adult phase, the mites were observed every $24 \mathrm{~h}$, to determine the reproductive parameters. The intrinsic rate of natural increase $\left(\mathrm{r}_{\mathrm{m}}\right)$ was 0.11 female/ female/day; resulting in a finite rate of increase of $1.11(\lambda)$. The net reproductive rate $\left(\mathrm{R}_{0}\right)$ was 7.1 females/generation, with a mean generation time ( $T$ ) 18.6 days. The results show that $T$. putrescentiae is a favorable food source for the development of I. zuluagai.
\end{abstract}

KEY WORDS: Citrus, Brevipalpus phoenicis, predatory mite, Astigmata

RESUMO - Iphiseiodes zuluagai Denmark \& Muma é um importante predador de Brevipalpus phoenicis (Geijskes) em citros no Brasil. O emprego de Tyrophagus putrescentiae (Schrank) como fonte de alimento para $I$. zuluagai em criações de laboratório foi investigado a $25,5 \pm 0,5^{\circ} \mathrm{C}, 88 \pm 7 \% \mathrm{UR}$ e fotofase de $12 \mathrm{~h}$. Inicialmente os níveis de oviposição do predador alimentado com ovos, estágios pósembrionários mortos ou estágios pós-embrionários vivos de T. putrescentiae foram avaliados durante 10 dias. A taxa diária de oviposição foi de 1,3 ovo/ fêmea quando estas foram alimentadas com ovos de T. putrecentiae; $0,7 \mathrm{ovo} /$ fêmea quando estas foram alimentadas com estágios pós-embrionários mortos e cerca de $0,2 \mathrm{ovo} /$ fêmea quando alimentadas com estágios pós-embrionários vivos. Posteriormente, elaborou-se a tabela de vida de I. zuluagai, oferecendo-se como alimento ovos de T. putrescentiae. Os estágios imaturos foram observados a cada $8 \mathrm{~h}$, para determinar a duração correspondente. $\mathrm{Na}$ fase adulta, os ácaros foram observados a cada $24 \mathrm{~h}$, para se determinar os parâmetros reprodutivos. A capacidade de aumento populacional $\left(\mathrm{r}_{\mathrm{m}}\right)$ foi de 0,11 fêmea/ fêmea/ dia; resultando em uma razão finita de aumento de $1,11(\lambda)$. A taxa líquida de reprodução $\left(\mathrm{R}_{0}\right)$ foi de 7,1 fêmeas/geração, com um tempo de geração de 18,6 dias. Os resultados obtidos mostram que T. putrescentiae é uma fonte de alimento favorável ao desenvolvimento de I. zuluagai.

PALAVRAS-CHAVE: citros, Brevipalpus phoenicis, ácaro predador, Astigmata

As plantas cítricas são atacadas por diversas pragas, sendo o ácaro Brevipalpus phoenicis (Geijskes) considerado espécie importante na cultura por transmitir o vírus causador da leprose dos citros (Kitajima et al. 1972).

O controle desse ácaro é regularmente feito através de acaricidas, porém o surgimento de populações resistente a esses produtos tem nos últimos anos comprometido a eficiência do controle químico (Omoto 1995; Omoto et al. 2000, Campos \& Omoto 2002).

O potencial dos ácaros predadores, principalmente Phytoseiidae, para o controle biológico ácaros fitófagos já é conhecido há bastante tempo (McMurtry \& Croft 1997, Moraes 2002). Gerson et al. (2003) listaram 28 espécies de ácaros utilizados em controle biológico, 
das quais 19 pertencem à família Phytoseiidae. Dentre os fitoseídeos, Phytoseiulus persimilis Athias-Henriot, Neoseiulus cucumeris (Oudemans), Neoseiulus californicus (McGregor) e Galendromus occidentalis (Nesbitt) são as mais comercializadas, principalmente para o controle de Tetranychus urticae (Koch). No Brasil, N. californicus tem sido liberado em macieira para o controle de Panonychus ulmi (Koch) com bastante sucesso (Monteiro 2002).

Dos predadores mais comuns e abundantes em citros, destaca-se Iphiseiodes zuluagai Denmark \& Muma (Chiavegato 1980, Bittencourt \& Cruz 1988, Sato 1994), sendo relatado em vários estados do Brasil (Moraes 2002), freqüentemente associado a B. phoenicis (Sato et al. 1994, Raga et al. 1996, Reis et al. 2000). Em laboratório, I. zuluagai mostrou-se bastante eficiente como predador de $B$. phoenicis (Reis et al. 2003), podendo ainda se alimentar de pólen e substâncias açucaradas (Yamamoto \& Gravena 1996, Reis et al. 1998), características desejáveis para um agente de controle biológico, pois propicia sua criação sobre fontes de alimento alternativas.

Alguns ácaros predadores são criados comercialmente em laboratório usando-se como presas ácaros da ordem Astigmata. Dentre os mais conhecidos, destacam-se Neoseiulus barkeri Hughes, criado sobre Acarus siro L. (Zhang 2003) e Stratiolelaps miles (Berlese), sobre T. putrescentiae (Steiner et al. 1999).

A possibilidade de desenvolvimento de um método de produção massal de $I$. zuluagai sobre $T$. putrescentiae dá perspectiva para a utilização desse predador para liberação em pomares orgânicos de citros, em sistemas de produção orgânica de citros. O objetivo deste trabalho foi avaliar o potencial de T. putrescentiae como fonte alternativa para $I$. zuluagai visando ao futuro desenvolvimento de um método para produção massal.

\section{Material e Métodos}

Este estudo foi conduzido no laboratório de Acarologia do Departamento de Entomologia, Fitopatologia e Zoologia Agrícola da ESALQ-USP, a $25,5 \pm 0,5^{\circ} \mathrm{C}, 88 \pm 7 \%$ de umidade relativa e fotofase de $12 \mathrm{~h}$. Como alimento do predador, foram avaliados diferentes estágios do ácaro $T$. putrescentiae.

Criação de $T$. putrescentiae. Os ácaros utilizados para iniciar a criação foram obtidos da criação massal mantida em laboratório. Um recipiente de vidro cilíndrico com volume de para $100 \mathrm{ml}$ foi utilizado como unidade para a criação. Para ventilação, foi aberto um orifício de $3 \mathrm{~cm}$ de diâmetro na tampa, o qual foi fechado com tela de malha fina. Como alimento, foram fornecidas semanalmente $2,4 \mathrm{~g}$ de ração semi-úmida para cães (DeliDog Purina $\left.{ }^{\circledR}\right)$. Trinta dias mais tarde, retirou-se uma amostra de $1,0 \mathrm{~g}$ do substrato contido nas unidades que continham ovos e formas móveis do ácaro e partículas de ração. Com o auxílio de um jato de água, esse material foi peneirado, utilizando-se uma peneira de 200 mesh sobreposta a outra de 500 mesh. Apenas o material retido na peneira de 500 mesh, composto de ovos e partículas finas de ração, foi utilizado para criação do predador. Esse material foi mantido a $-8^{\circ} \mathrm{C}$ por 40 min para minimizar a eclosão de larvas, sendo em seguida utilizado para a criação dos predadores.

Criação de manutenção de $I$. zuluagai. A criação de $I$. zuluagai foi iniciada a partir de ácaros recém-coletados em citros do Setor de Zoologia do Departamento de Entomologia, Fitopatologia e Zoologia Agrícola da ESALQ-USP. Estes foram mantidos em unidades de criação confeccionadas com placas de resina (Paviflex ${ }^{\circledR}$ ) de 10 x $15 \mathrm{~cm}$, colocadas sobre um pedaço de espuma de polietileno de $2 \mathrm{~cm}$ de altura, permanentemente umedecido com água destilada, no interior de bandejas. As bordas de cada placa foram cobertas com tiras de algodão, para evitar a fuga dos ácaros. No centro de cada placa, fios de algodão foram colocados sob uma lamínula de microscopia $(2 \times 2 \mathrm{~cm})$, para servir de abrigo e local para oviposição. Como alimento, foram utilizados ovos de T. putrescentiae obtidos da forma indicada no item anterior. A criação foi observada diariamente, adicionando-se mais alimento quando necessário.

Oviposição em diferentes estágios de desenvolvimento de T. putrescentiae. As fases de ovo e estágios pós-embrionários da presa foram separadas utilizando-se uma adaptação do método Jenkis (1964), comumente utilizado para extração de nematóides de raízes de plantas. Para tanto, retirou-se da criação de T. putrescentiae uma amostra de $1,0 \mathrm{~g}$ do substrato, procedendo-se o peneiramento como anteriormente descrito. O material retido na peneira de 200 mesh foi transferido para um tubo de centrífuga, adicionando-se $1,0 \mathrm{~cm}^{3}$ de caolim, completando-se com água o peso para $100 \mathrm{~g}$. O mesmo procedimento foi adotado em relação ao material retido na peneira de 500 mesh. Em seguida, o material foi centrifugado por $5 \mathrm{~min}$ a $1.800 \mathrm{rpm}$, descartando-se o sobrenadante. Ao precipitado adicionou-se uma solução de sacarose (400 g de açúcar para $750 \mathrm{ml}$ de água) até o peso de $100 \mathrm{~g}$. Em seguida o material foi centrifugado por mais $1 \mathrm{~min}$ a 1.800 $\mathrm{rpm}$. O sobrenadante de cada tubo que continha os ácaros foi vertido sobre uma peneira de 500 mesh, lavando-se o material com água destilada, para retirar o excesso de sacarose. Esse procedimento permitiu a separação eficiente dos ovos (inicialmente retidos na peneira de 500 mesh) e dos outros estágios pós-embrionários (inicialmente retidos na peneira de 200 mesh), das partículas de ração.

Para reduzir a eclosão das larvas, os ovos foram acondicionados em recipientes fechados e colocados a $-8^{\circ} \mathrm{C}$ por $40 \mathrm{~min}$, sendo depois mantidos até o final dos trabalhos em refrigerador a $5^{\circ} \mathrm{C}$. Os estágios pós-embrionários foram divididos em dois grupos, um acondicionado a $-8^{\circ} \mathrm{C}$, por $24 \mathrm{~h}$, para sua inativação, e outro confinado em arenas de placas de resina sem alimento, e imediatamente utilizado nos experimentos.

Cinqüenta fêmeas adultas de I. zuluagai, aparentemente sadias, foram transferidas da criação de manutenção para novas arenas e deixadas a ovipositar durante $12 \mathrm{~h}$. A partir da eclosão, as larvas dos predadores foram alimentadas com ovos inviáveis de T. putrescentiae até atingirem a fase adulta, sendo então utilizados no testes. Cada fêmea recémemergida foi transferida para uma unidade experimental (2,6 cm de diâmetro e $1 \mathrm{~cm}$ de altura, contendo um disco de 
folha de feijão-de-porco, Canavalia ensiformis D.C., de 2,5 $\mathrm{cm}$ de diâmetro colocado sobre um disco de papel filtro de mesmo diâmetro umedecido com água destilada). Em cada unidade, foi também colocado um macho retirado da criação de manutenção, que foi mantido juntamente com a fêmea durante todo o teste. Cada casal foi diariamente alimentado com ovos, estágios pós-embrionários mortos ou estágios pós-embrionários vivos.

As unidades foram fechadas com filme de plástico transparente de PVC (Magipack ${ }^{\circledR}$ ), para evitar a fuga dos predadores, e observadas diariamente sob microscópio estereoscópico, anotando-se o número de ovos postos por fêmea de I. zuluagai durante 11 dias. Para efeito de cálculo, o primeiro dia foi descartado devido à possível influência da alimentação anterior. A oviposição média em cada tratamento foi comparada pelo teste de Tukey $(\mathrm{P}<0,05)$.

Tabela de vida de fertilidade. Pelo mesmo processo descrito anteriormente, foram obtidos 50 ovos de I. zuluagai com idade variando de 0 até $12 \mathrm{~h}$, que foram isolados em unidades experimentais semelhantes às descritas anteriormente. As unidades foram observadas a cada $8 \mathrm{~h}$ sob microscópio estereoscópico. A partir da emergência das larvas, os predadores foram alimentados com ovos de T. putrescentiae. Colocou-se em cada unidade aproximadamente $0,02 \mathrm{~g}$ de ovos. O alimento consumido foi reposto diariamente. A cada cinco dias, os discos de folha de feijão-de-porco foram substituídos. A ocorrência das ecdises foi determinada pela presença das exúvias.

À medida que emergiam, os adultos foram sexados, formando-se os casais. Devido ao maior número de fêmeas do que machos, foi necessário utilizar machos da criação de manutenção para formar casais com as fêmeas restantes. Os machos mortos foram substituídos por outros retirados da criação de manutenção. As unidades foram examinadas diariamente, quantificando-se o número de ovos do predador e transferindo-os para outras unidades, para a obtenção de adultos e a determinação da razão sexual. As observações da geração parental seguiram-se até a morte das fêmeas. Com a determinação dos diferentes parâmetros das fases imatura e adulta, foi elaborada uma tabela de vida (Birch 1948), corrigida pela fórmula de Euler (Meyer et al. 1986).

\section{Resultados}

Oviposição em diferentes estágios de desenvolvimento de T. putrescentiae. A maior oviposição foi registrada quando $I$. zuluagai foi alimentada com ovos da presa de $T$. putrescentiae, seguida dos estágios pós-embrionários mortos e por fim, dos estágios pós-embrionários vivos (Tabela 1).

O baixo nível de oviposição do predador sobre estágios pós-embrionários vivos sugere a incompatibilidade entre $I$. zuluagai e alguns ou todos os estágios de $T$. putrescentiae. Apesar de não ter sido verificado nenhum tipo de comportamento defensivo da presa, muitas vezes ao aproximar-se da presa viva, I. zuluagai recuava rapidamente após tocar as setas desta. Entretanto, em certas ocasiões, principalmente no caso das fêmeas recém-emergidas, o predador imobilizava a presa de modo que ela ficasse com a
Tabela 1. Oviposição média ( \pm EP) diária de $I$. zuluagai alimentada com ovos, estágios pós-embrionários mortos (E.P.M.) e estágios pós-embrionários vivos (E.P.V.) de $T$. putrescentiae, $25,5 \pm 0,5^{\circ} \mathrm{C}, 88 \pm 7 \%$ de umidade relativa e $12 \mathrm{~h}$ de fotofase $(\mathrm{n}=20)$.

\begin{tabular}{lc}
\hline $\begin{array}{l}\text { Fase de desenvolvimento do } \\
\text { hospedeiro }\end{array}$ & Número de ovos/dia \\
\hline Ovo & $13,2 \pm 1,04 \mathrm{a}$ \\
E.P.M. & $7,0 \pm 0,30 \mathrm{~b}$ \\
E.P.V. & $0,15 \pm 0,08 \mathrm{c}$ \\
\hline
\end{tabular}

Médias seguidas de mesma letra não diferem significativamente pelo teste de Tukey $(\mathrm{P}<0,05)$.

face ventral do idiossoma voltada para cima, e em seguida, iniciava a predação.

Tabela de vida de fertilidade. A duração de cada estágio imaturo foi de aproximadamente um dia (Tabela 2). Cerca de $20,4 \%$ das larvas eclodiram em menos de $12 \mathrm{~h}, 24,2 \%$ entre $16 \mathrm{~h}$ e $24 \mathrm{~h}, 21,9 \%$ entre $24 \mathrm{~h}$ e $32 \mathrm{~h}, 29,5 \%$ entre $32 \mathrm{~h}$ e $40 \mathrm{~h}$ e apenas $4 \%$ acima de $40 \mathrm{~h}$. O estágio larval foi ligeiramente mais curto que os demais. A duração total de ovo a adulto foi aproximadamente quatro dias. A sobrevivência de ovo a adulto foi alta, atingindo $92 \%$, observando-se mortalidade apenas no estágio larval.

O período de pré-oviposição foi relativamente longo e ligeiramente maior que o período de pós-oviposição, correspondendo a cerca de $20 \%$ do período de oviposição (Tabela 2).

A fecundidade média foi de 16,6 ovos/fêmea. A oviposição média diária aumentou rapidamente, atingindo seu pico próximo do décimo dia, decrescendo também rapidamente a partir do décimo quinto dia, quando se estabilizou até cerca do vigésimo terceiro dia, reduzindo-se outra vez rapidamente (Fig. 1). A oviposição média diária para todo o período de

Tabela 2. Duração ( \pm EP) em dias dos estágios/fases de I. zuluagai alimentada com ovos mortos de T. putrescentiae, $25,5 \pm 0,5^{\circ} \mathrm{C}, 88 \pm 7 \%$ de umidade relativa e $12 \mathrm{~h}$ de fotofase.

\begin{tabular}{|c|c|c|c|}
\hline Estágios/fases & $\mathrm{n}$ & Duração & Sobrevivência \\
\hline Ovo & 50 & $1,1 \pm 0,08$ & 100 \\
\hline Larva & 50 & $0,7 \pm 0,03$ & 92 \\
\hline Protoninfa & 46 & $1,1 \pm 0,06$ & 100 \\
\hline Deutoninfa & 46 & $1,1 \pm 0,05$ & 100 \\
\hline Ovo-adulto & 46 & $3,9 \pm 0,08$ & 92 \\
\hline Pré-oviposição & 33 & $3,8 \pm 0,26$ & - \\
\hline Oviposição & 33 & $18,0 \pm 1,18$ & - \\
\hline Pós-oviposição & 33 & $3,2 \pm 0,23$ & - \\
\hline Longevidade $q$ & 33 & $25,4 \pm 1,21$ & - \\
\hline Longevidade $\widehat{\partial}$ & 16 & $8,0 \pm 0,47$ & - \\
\hline
\end{tabular}


oviposição foi de 0,9 ovo/fêmea/dia, sendo a razão sexual $67 \%$ de fêmeas. Com os dados de fecundidade, fertilidade, longevidade e razão sexual, elaborou-se a tabela de vida de fertilidade. A partir desta estimou-se que nas condições deste estudo a população de I. zuluagai apresentou um crescimento populacional de aproximadamente 7,1 vezes $\left(\mathrm{R}_{\mathrm{o}}\right)$ a cada 19 dias $(\mathrm{T})$, que corresponde ao aumento populacional diário da ordem de $11 \%(\lambda=1,11)$, com produção de 0,11 fêmea por fêmea por dia $\left(\mathrm{r}_{\mathrm{m}}\right)$.

A longevidade das fêmeas foi em média de 25 dias, enquanto que os machos apresentaram longevidade média de aproximadamente oito dias.

\section{Discussão}

Os valores satisfatórios de oviposição e de capacidade de aumento populacional observado no presente estudo sugerem que $T$. putrescentiae é uma presa adequada para o desenvolvimento de I. zuluagai. O período de incubação foi bastante curto diferindo do observado para outros fitoseídeos. A eclosão de larvas poucas horas após a postura pode ocorrer porque as fêmeas retêm os ovos no interior do idiossoma, aproximando-se de uma ovoviviparidade, como sugerido por Reis et al. (1998). Abbou-Setta et al. (1991) observaram larvas bem desenvolvidas dentro de ovos no interior do idiossoma de fêmeas de outra espécie do mesmo gênero, Iphiseiodes quadripilis (Banks). De acordo com os autores, esse fenômeno proporciona melhor chance de sobrevivência dos descendentes, o que poderia ser uma vantagem deste em relação a outros predadores. Esse aspecto poder contribuir para explicar a predominância desse predador nas plantas cítricas em períodos de baixa umidade relativa do ar, pelo fato de haver um encurtamento na fase de ovo, mais suscetível ao efeito do clima. Sanderson \& McMurtry (1984) verificaram que fêmeas de Phytoseius hawaiiensis Prasad aparentemente mantinham o ovo no interior do corpo, permitindo seu desenvolvimento interno, também quase a ponto de uma ovoviviparidade. Amitai \& Grinberg (1971) estudaram a possível viviparidade Paragigagnathus tamaricis Amitai \& Grinberg, verificando que fêmeas adultas apresentavam larvas em seu interior.

A duração relativamente curta do tempo de incubação parece também estar relacionada ao longo período de préoviposição de I. zuluagai, observado no presente estudo. Reis et al. (1998) verificaram que esse predador apresentou período de pré-oviposição de quatro dias quando alimentado com pólen de mamoneira (Ricinus communis L.), enquanto Yamamoto \& Gravena (1996) observaram um período de 5,5 dias para o mesmo parâmetro, com o mesmo alimento.

A oviposição média diária observada foi ligeiramente superior àquela encontrada por Reis et al. (1998), no entanto, ficou bem abaixo da observada por Yamamoto \& Gravena (1996). Essas diferenças podem estar relacionadas à variabilidade genética das populações estudadas (Reis et al. 1998), ou ao tipo de alimento oferecido.

Reis \& Haddad (1997) determinaram longevidade média de 23 dias para fêmeas de I. zuluagai e verificaram que machos acasalados apresentaram longevidade média de oito dias, enquanto machos não acasalados apresentaram longevidade média de 15 dias. Yamamoto \& Gravena (1996) verificaram que para fêmeas do mesmo ácaro a longevidade média foi de 35 dias quando alimentadas com pólen de $R$. communis e 28,5 dias com pólen de taboa, Tipha angustifolia $\mathrm{L}$.

Os parâmetros da tabela de vida sugeriram que a presa oferecida supriu as necessidades nutricionais do predador, próximo do que foi obtido por Reis et al. (1998), diferindo, entretanto de Yamamoto \& Gravena (1996), que obtiveram melhores resultados. As diferenças observadas com outros autores, neste estudo, podem estar relacionadas com aspectos fisiológicos das populações estudadas, além do tipo de alimento oferecido.

Os hábitos alimentares de fitoseídeos são bastante variáveis, havendo espécies que são predadares obrigatórias, outras que são predadoras facultativas, podendo sobreviver alimentando-se de fungos, pólen, substâncias açucaradas, e

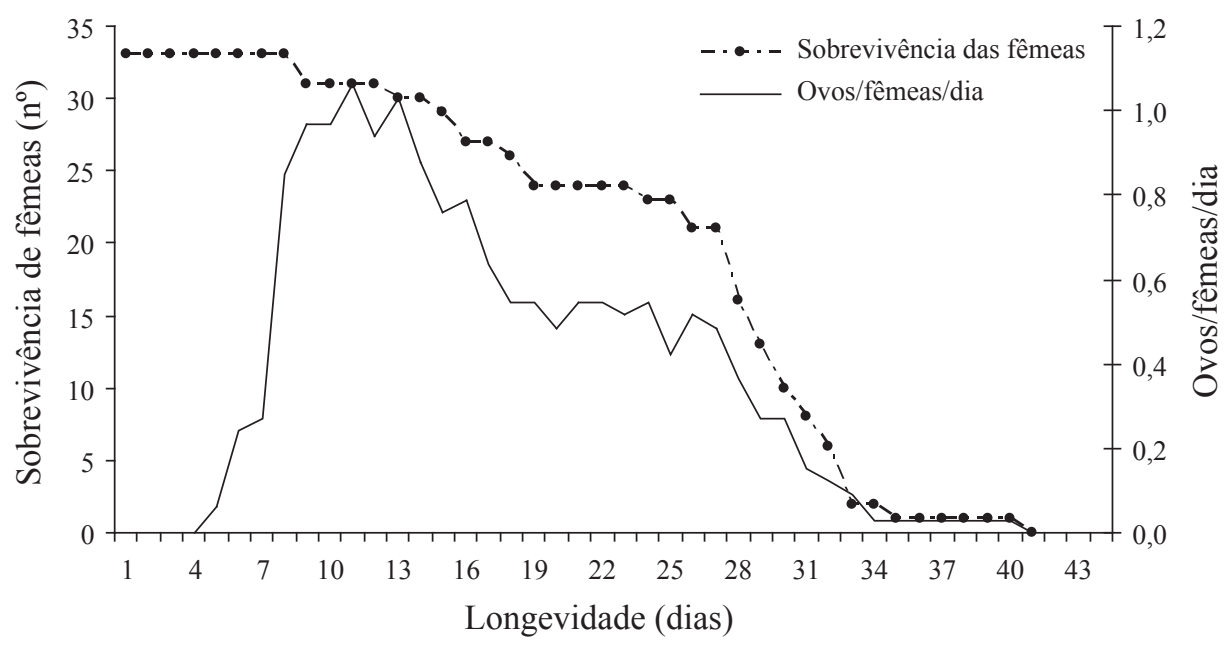

Fig. 1. Sobrevivência e oviposição média diária de fêmeas de $I$. zuluagai, alimentadas com ovos inativados de $T$. putrescentiae. 
ainda outras que são predadoras facultativas que têm o pólen como principal fonte de alimento (Sanderson \& McMurtry 1984, Bounfour \& McMurtry 1987, Croft et al. 1998).

Muma (1971) sumariou os diferentes efeitos de fontes alimentares sobre 11 espécies de Phytoseiidae, verificando grande variação quanto à adequação das fontes para os predadores. Dentre os predadores, o autor observou que $I$. quadripilis aceitou uma vasta gama de fontes alimentares, característica dos ácaros do grupo III (McMurtry \& Croft 1997). Dentre as fontes de alimento os Acaridae se comportaram como inadequados a ótimos. Além dos Acaridae, Muma (1971) mostrou que I. quadripilis também pode se desenvolver sobre ácaros das famílias Tarsonemidae, Tetranychidae e Tydeidae. $\mathrm{O}$ autor não detalhou quais as espécies dessas famílias seriam utilizadas como fonte alimentar, mas sugeriu que os congêneres do predador podem ter a mesma diversidade alimentar aparentemente apresentada por I. quadripilis.

$\mathrm{O}$ fato de $I$. zuluagai ter se desenvolvido melhor quando alimentada com ovos do que com as outras formas oferecidas está de acordo com o encontrado para outros fitoseídeos. O estágio preferencial da presa para predação varia bastante dentre os ácaros predadores. Blackwood et al. (2001) avaliaram a preferência para alimentação de 13 espécies de ácaros predadores, por ovos e larvas de T. urticae, concluindo que os ácaros mais especialistas preferiram alimentar-se de ovos, enquanto que os generalistas preferiram as larvas. Por outro lado, Steiner et al. (2003) verificaram que Typhlodromips montdorensis (Schicha) consumiu indiscriminadamente ovos e estágios pós-embrionários de T. putrescentiae.

I. zuluagai é um predador capaz de explorar diversas fontes de alimento (Yamamoto \& Gravena 1996; Reis et al. 1998, 2003), podendo ser classificado como predador tipo III (McMurtry \& Croft 1997). O fato de I. zuluagai alimentar-se de Astigmata amplia o conhecimento sobre as fontes alimentares disponíveis para ele. Este trabalho constitui o primeiro relato desse predador alimentando-se de T. putrescentiae.

T. putrescentiae é encontrada em diversos tipos de ambiente, mas é apenas raramente encontrado sobre plantas. Por outro lado, Iphiseiodes sp. tem sido até o momento constatada apenas em plantas. Isso sugere que na natureza esses organismos provavelmente nunca se encontrem. De qualquer maneira, os resultados obtidos no presente estudo mostraram que T. putrescentiae é uma presa aceita por $I$. zuluagai permitindo não apenas sua sobrevivência, mas também sua reprodução a níveis comparáveis com aqueles proporcionados por outras fontes de alimento avaliadas por outros autores.

$\mathrm{O}$ fato de I. zuluagai ter se desenvolvido satisfatoriamente sobre T. putrescentiae abre uma perspectiva de que o predador possa ser produzido mais facilmente em quantidades suficientes para liberação em campo. A criação poderá ser mantida com uma mistura de ovos e estágios pós-embrionários mortos, entretanto, o método de separação desses estágios do estágio adulto precisa ser mais bem definido, pois o que foi utilizado neste trabalho requer um aparelhamento que a princípio dificulta o processo de criação do predador. Assim, avaliar outras espécies de Acaridae pode ser uma alternativa viável para o estabelecimento de uma metodologia para a criação massal de I. zuluagai.

\section{Referências}

Abbou-Setta, M.M., M.S. Nawar \& C.C. Childers. 1991. Description of post-embryonic stages of Iphiseiodes quadripilis, a predatory mite on Florida citrus (Acari: Phytoseiidae). Int. J. Acarol. 17: 241-248.

Amitai, S. \& T. Grinberg. 1971. Description of a new phytoseiid genus and species (Acarina: Phytoseiidae) from Israel. Israel J. Entomol. 6: 327-335.

Birch, L.C. 1948. The intrinsic rate of natural increase of an insect population. J. Anim. Ecol. 17: 15-26.

Bittencourt, M.A.L. \& F.Z. da Cruz. 1988. Toxicidade de produtos químicos sobre ácaros predadores (Acari: Phytoseiidae) em citros. An. Soc. Entomol. Brasil 17: 249-261.

Blackwood, J.S., P. Shausberger \& B.A. Croft. 2001. Prey-stage preference in generalist and specialist phytoseiid mites (Acari: Phytoseiidae) when offered Tetranychus urticae (Acari: Tetranychidae) eggs and larvae. Environ. Entomol. 30: 1103-1111.

Bounfour, M. \& J.A. McMurtry. 1987. Biology and ecology of Euseius scutalis (Athias-Henriot) (Acarina: Phytoseiidae). Hilgardia 55: 1-23.

Campos, F.J. \& C. Omoto. 2002. Resistence to hexythiazox in Brevipalpus phoenicis (Acari: Tenuipalpidae) from Brazilian citrus. Exp. Appl. Acarol. 26: 243-251.

Chiavegato, L.G 1980. Ácaros da cultura do citros, p.469-491. In O. Rodrigues \& F. Viégas (coords.) Citricultura brasileira. Fundação Cargill, Campinas, 739p.

Croft, B.A., J.A. McMurtry \& H. -K. Luh. 1998. Do literature records of predation reflect food specialization and predation types among phytoseiid mites (Acari: Phytoseiidae)? Exp. Appl. Acarol. 22: 467-480.

Gerson, U., R.L. Smiley \& R. Ochoa. 2003. Mites (Acari) for pest control. Oxford, Blackwell Publishing, 539p.

Jenkins, W.R. 1964. A rapid centrifugal-flotation technique for separating nematodes from soil. Plant Dis. Rep. 48: 692.

Kitajima, E.W., G.W. Müller, A.S. Costa \& V. Yuki. 1972. Short rod-like particles associated with citrus leprosis. Virology 50: 254-258.

McMurtry, J.A. \& B.A. Croft. 1997. Life-styles of phytoseiid mites and their roles in biological control. An. Rev. Entomol. 42: 291-321.

Meyer, J.S., C.G. Igersoll, L.L. MacDonald \& M.S. Boyce. 1986. Estimating uncertainty in population growth rates: Jackknife vs. bootstrap techniques. Ecology 67: 1156-1166.

Monteiro, L.B. 2002. Criação de ácaros fitófagos e predadores: Um caso de produção de Neoseiulus californicus por produtores de maçã, p.351-365. In J.R.P. Parra, P.S.M. Botelho, B.S. CorrêaFerreira \& J.M.S. Bento (eds.), Controle biológico no Brasil - parasitóides e predadores. Barueri, Manole, 609p.

Moraes, G. J. de. 2002. Controle biológico de ácaros fitófagos com ácaros predadores, p.225-237. In J.R.P. Parra, P.S.M. Botelho, 
B.S. Corrêa-Ferreira \& J.M.S. Bento (eds.), Controle biológico no Brasil - parasitóides e predadores, Barueri, Manole, 609p.

Muma, M.H. 1971. Food habitats of phytoseiid (Acarina: Mesostigmata) including common species on Florida citrus. Fla Entomol. 54: 21-34.

Omoto, C. 1995. Resistência de Brevipalpus phoenicis (Acari: Tenuipalpidae) aos produtos químicos na citricultura, p.179188. In C.A.L. Oliveira. \& L.C. Donadio (eds.), Leprose dos citros. Jaboticabal, Funep, 219p.

Omoto, C., E.B. Alves \& P.C. Ribeiro. 2000. Detecção e monitoramento da resitência de Brevipalpus phoenicis (Geijskes) (Acari: Tenuipalpidae) ao dicofol. An. Soc. Entomol. Brasil 29: 757-764.

Raga, A., M.E. Sato, L.C. Cerávolo \& A.C. Rossi. 1996. Distribuição de ácaros predadores (Phytoseiidae) em laranjeira (Citrus sinensis L. Osbeck). Rev. Ecossist. 21: 23-25.

Reis, P.R., E.O. Souza, A.V. Teodoro \& M. Pedro Neto. 2003. Effect of prey density on the functional and numerical responses of two species of predaceous mites (Acari: Phytoseiidae). Neotrop. Entomol. 32: 461-467.

Reis, P.R., L.G. Chiavegato \& E.B. Alves. 1998. Biologia de Iphiseiodes zuluagai Denmark \& Muma (Acari: Phytoseiidae). An. Soc. Entomol. Brasil 27: 185-191.

Reis, P.R. L.G. Chiavegato, E.B. Alves \& E.O. Sousa. 2000. Ácaros da família Phytoseiidae associados aos citros no município de Lavras, Sul de Minas Gerais. An. Soc. Entomol. Brasil 29: 95-104.
Reis, P.R. \& M.L. Haddad. 1997. Distribuição de Weibull como modelo de sobrevivência de Iphiseiodes zuluagai Denmark \& Muma (Acari: Phytoseiidae). An. Soc. Entomol. Brasil 26: 441-444.

Sanderson, J.P. \& J.A. McMurtry. 1984. Life history studies of the predaceous mite Phytoseius hawaiiensis. Entomol. Exp. Appl. 35: 227-234.

Sato, M.E., A. Raga., L.C., Cerávolo, A.C. Rossi \& M.R. Potenza. 1994. Ácaros predadores em pomar cítrico de Presidente Prudente, estado de São Paulo. An. Soc. Entomol. Brasil 23: 435-441.

Steiner, M., S. Goodwin \& T. Wellham. 1999. A simplified rearing method for Stratiolaelaps (Hypoaspis) miles (Acari: Laelapidae). IOBC/WPRS Bull. 22: 241-242.

Steiner, M.Y. 2003. Biological studies of the Australian predatory mite Typhlodromips montdorensis (Schicha) (Acari: Phytoseiidae), a potential biocontrol agent for western flower thrips, Frankliniella occidentalis (Pergande) (Thysanoptera: Thripidae). Aust. J. Entomol. 42: 124-130.

Yamamoto, P.T. \& S. Gravena. 1996. Influência de temperatura e fontes de alimento no desenvolvimento e oviposição de Iphiseiodes zuluagai Denmark \& Muma (Acari: Phytoseiidae). An. Soc. Entomol. Brasil 25: 109-115.

Zhang, Z.Q. 2003. Mites of greenhouses: Identification, biology and control. Wallingford, CABI Publishing, 244p.

Received 03/IV/07. Accepted 14/IV/08. 